U N IVERSITY OF COPENHAGEN

\title{
Decreasing Relative Risk Premium
}

Hansen, Frank

Publication date:

2006

Document version

Publisher's PDF, also known as Version of record

Citation for published version (APA):

Hansen, F. (2006). Decreasing Relative Risk Premium. Department of Economics, University of Copenhagen. 


\title{
Decreasing relative risk premium
}

\author{
Frank Hansen
}

February 222007

\begin{abstract}
We consider the risk premium $\pi$ demanded by a decision maker with present wealth $x$ in order to be indifferent between obtaining a new level of wealth $y_{1}$ with certainty, or to participate in a lottery which either results in unchanged wealth $x$ or a level of wealth $y_{2}>y_{1}$. We then define the relative risk premium $\lambda$ as the quotient between $\pi$ and the increase in wealth $y_{1}-x$ which the decision maker puts on the line by choosing the lottery in place of receiving $y_{1}$ with certainty. We study preferences such that the relative risk premium is a decreasing function of present wealth, and we determine the corresponding class of utility functions which has several attractive properties and contains functions frequently used in the literature, including the power utility functions. The functions in the class are automatically continuously differentiable, and we characterize them in several ways. Decreasing relative risk premium in the small implies decreasing relative risk premium in the large, and decreasing relative risk premium everywhere implies risk aversion. We finally introduce the notion of partial risk neutral preferences on binary lotteries and show that partial risk neutrality is equivalent to preferences with decreasing relative risk premium.
\end{abstract}

JEL classification: D8 and G12.

Key words: Expected utility theory, relative risk premium, preferences on lotteries, partial risk neutrality.

\section{Introduction}

In the theory of Morgenstern and von Neumann (with later contributions) preferences over lotteries are resolved by calculating expected utility from a utility function $u$ which is unique up to the composition with increasing affine transformations. The assumptions of greed and risk aversion are then 
characterized by the requirements that $u$ is non-decreasing and concave. It is well-known [9] that some utility functions within this class imply an implausible fast depreciation of marginal utility as a function of wealth. This applies for example to functions with constant absolute risk aversion.

Consider a decision maker with wealth $x$ who is given the choice between obtaining an alternative level of wealth $y_{1}$ with certainty, or to participate in a lottery which will result in either unchanged wealth $x$ or a level of wealth $y_{2}$ strictly bigger than $y_{1}$.

The problem arises in situations where the decision maker is confronted with a choice between two mutually exclusive possibilities. Either engage in an activity which results in wealth $y_{1}$ with certainty, or try an alternative activity which, if successful, gives a higher level of wealth $y_{2}>y_{1}$ but may fail to materialize and then leaves the decision maker with the present level of wealth $x$ unchanged.

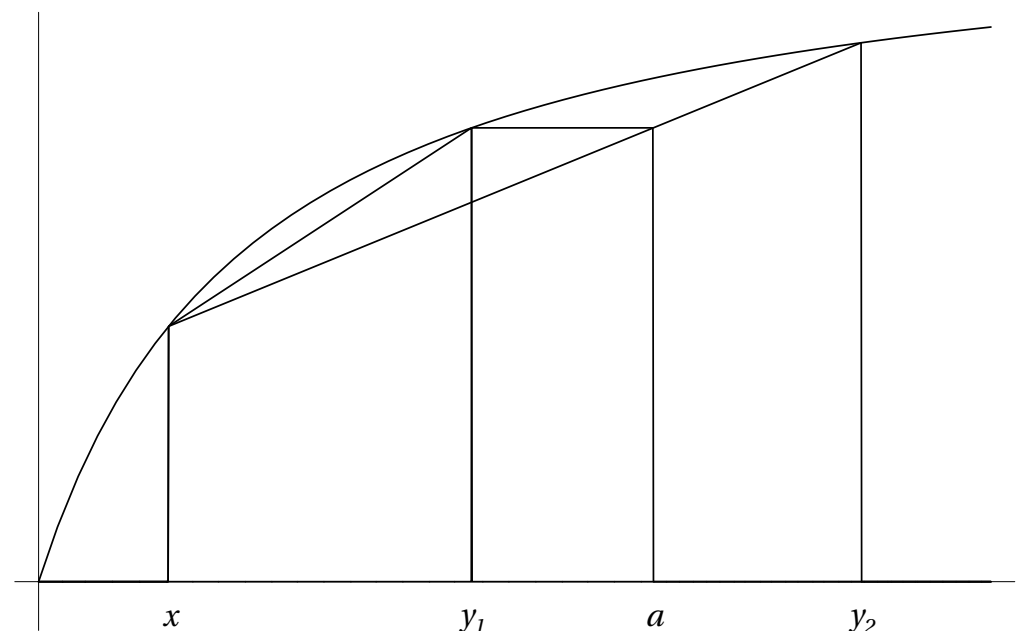

Let $a=a\left(x, y_{1}, y_{2}\right)$ be the expected level of wealth in the lottery between $x$ and $y_{2}$ which gives the same expected utility as obtaining $y_{1}$ with certainty. The agent is thus indifferent between obtaining the level of wealth $y_{1}$ for sure or participate in a lottery between $x$ and $y_{2}$ giving the expected wealth $a$. We define for $y_{1}<y_{2}$ the relative risk premium

$$
\lambda(x)=\frac{a-y_{1}}{y_{1}-x} \quad x \neq y_{1}
$$

as the quotient between the risk premium $\pi=a-y_{1}$ divided by the opportunity cost $y_{1}-x$ which the agent puts on the line by choosing the lottery in place of $y_{1}$ with certainty. We note that $a$ and therefore $\lambda$ only depend on $x, y_{1}, y_{2}$ and the values of the utility function in these three points. It is by construction a reflection of the agent's preferences and is therefore left 
invariant under affine transformations of the utility function, which may also be verified directly.

The discussion above is made under the implicit assumptions that the utility function is concave and $x<y_{1}$ such that the risk premium is positive and the certainty equivalent is placed between $y_{1}$ and $y_{2}$. But this is done only to facilitate the reader's perception, and no such restrictions are to be imposed. If for example the utility function is convex, then the risk premium is negative.

Definition 1.1. Let $u$ be an increasing utility function defined in an open interval $I=(\alpha, \beta)$. We say that u represents decreasing relative risk premium, if the relative risk premium $\lambda(x)$, for arbitrary $y_{1}<y_{2}$ in $I$, is a decreasing function in $x \in I \backslash\left\{y_{1}, y_{2}\right\}$.

We develop a very easy-to-use criterion for determining whether a utility function represents decreasing relative risk premium, cf. Theorem 2.4 (iii), and it follows that often used utility functions like

$$
u(x)=x^{\alpha}, \quad u(x)=\frac{x}{x+\lambda}, \quad u(x)=\log x,
$$

where $\alpha \in[0,1]$ and $\lambda \geq 0$ and their compositions like for example

$$
u(x)=\left(\frac{x}{x+\lambda}\right)^{\alpha} \text { and } u(x)=\log \left(\frac{x}{x+\lambda}\right)
$$

all represent decreasing relative risk premium. The property is thus satisfied in many situations already considered by economists. But there are also functions, used in for example financial theory, which are not in the class. This applies most notably to functions like $u(x)=1-\exp (-x)$ with constant absolute risk aversion.

The notion of relative risk premium focuses more on the terminal level of wealth than on the pay-off in the lottery. As will be demonstrated later, it has a local version which makes it possible to compare it with Pratt's measure [7] of absolute risk aversion. The two notions are different, but decreasing relative risk premium implies decreasing absolute risk aversion. The function $u(x)=1-\exp (-\sqrt{x})$ does not represent decreasing relative risk premium, but it is increasing, concave and the absolute risk aversion is tending to zero as $x$ approaches infinity.

In Section 3 we consider lotteries of the form $(\tilde{x}, p)$ with outcomes $\left(x_{1}, x_{2}\right)$, where $p$ is the probability of obtaining $x_{1}$. To every set of intertwined outcomes $x_{1}<y_{1}<x_{2}<y_{2}$ we introduce in equation (5) a mapping $p \rightarrow q(p)$, depending only on the outcomes, of the unit interval into itself. The expected 
outcomes of the lotteries satisfy $E(\tilde{x}, p) \leq E(\tilde{y}, q)$ for every $p \in[0,1]$ and every $q \in[0, q(p)]$. We say that a decision maker is partially risk neutral if he prefers $(\tilde{y}, q)$ to $(\tilde{x}, p)$ for every $p \in[0,1]$ and every $q \in[0, q(p)]$, that is if the decision maker chooses as if he were risk neutral in these choice situations.

We finally obtain that a decision maker is partially risk neutral if and only if his preferences represent decreasing relative risk premium. This opens up the possibility of analyzing markets with highly inhomogeneous investors.

\section{Decreasing relative risk premium}

It is not at all obvious that a sum of two utility functions, each with decreasing relative risk premium, has decreasing relative risk premium, but we do note that the point-wise limit of utility functions with decreasing relative risk premium retains the property.

\subsection{Geometric description}

The expected level of wealth $a$ is calculated by setting

$$
u(x)+(a-x) \frac{u\left(y_{2}\right)-u(x)}{y_{2}-x}=u\left(y_{1}\right),
$$

therefore the risk premium

$$
\pi=a-y_{1}=\left(y_{1}-x\right)\left(\frac{\left[x, y_{1}\right]_{u}}{\left[x, y_{2}\right]_{u}}-1\right),
$$

where the divided difference $[t, s]_{u}$ is defined by setting

$$
[t, s]_{u}=\frac{u(t)-u(s)}{t-s} \quad t \neq s .
$$

The relative risk premium $\lambda$ is therefore given by

$$
\lambda(x)=\frac{\pi}{y_{1}-x}=\frac{\left[x, y_{1}\right]_{u}-\left[x, y_{2}\right]_{u}}{\left[x, y_{2}\right]_{u}},
$$

and it is the relative increase in slope of the line connecting $(x, u(x))$ and $\left(y_{1}, u\left(y_{1}\right)\right)$ in comparison with the line connecting $(x, u(x))$ and $\left(y_{2}, u\left(y_{2}\right)\right)$. 


\subsection{Various characterizations}

We begin by characterizing utility functions with decreasing relative risk premium in terms of their monotonicity properties when lifted to functions of two by two matrices. The characterization implies that a sum of utility functions, each with decreasing relative risk premium, retains the property. The notion of decreasing relative risk premium may therefore be used also in the study of aggregated demand.

Let $A=\left(a_{i j}\right)$ be a Hermitian two by two matrix with eigenvalues $\lambda$ and $\mu$ in the interval $I$. It can be written on the form

$$
A=\left(\begin{array}{ll}
a_{11} & a_{12} \\
a_{21} & a_{22}
\end{array}\right)=Q\left(\begin{array}{cc}
\lambda & 0 \\
0 & \mu
\end{array}\right) Q^{-1}
$$

where $Q$ is an orthogonal matrix. The functional calculus is defined by setting

$$
u(A)=Q\left(\begin{array}{cc}
u(\lambda) & 0 \\
0 & u(\mu)
\end{array}\right) Q^{-1}
$$

We say that $u$ is 2 -monotone in the interval $I$ if

$$
A \leq B \quad \Rightarrow \quad u(A) \leq u(B)
$$

for all Hermitian two by two matrices $A$ and $B$ with eigenvalues in $I$. Note that the order relation is defined by setting $A \leq B$ if $B-A$ is positive semi-definite.

Theorem 2.1. The relative risk premium $\lambda$ of an increasing function $u$ is decreasing if and only if $u$ is 2-monotone.

Proof. The relative risk premium is decreasing if and only if

$$
\frac{\left[x_{1}, y_{1}\right]_{u}}{\left[x_{1}, y_{2}\right]_{u}} \geq \frac{\left[x_{2}, y_{1}\right]_{u}}{\left[x_{2}, y_{2}\right]_{u}}
$$

or equivalently, that the determinant of the (non-symmetric) matrix

$$
\operatorname{det}\left(\begin{array}{ll}
{\left[x_{1}, y_{1}\right]_{u}} & {\left[x_{1}, y_{2}\right]_{u}} \\
{\left[x_{2}, y_{1}\right]_{u}} & {\left[x_{2}, y_{2}\right]_{u}}
\end{array}\right) \geq 0
$$

for $x_{1}<x_{2}$ and $y_{1}<y_{2}$. But Löwner's theorem [5] characterizes the 2monotone functions by exactly this condition.

QED 
Inspection of the reference reveals that condition (1) is only required for points in $I$ with $x_{1}<y_{1}<x_{2}<y_{2}$ and then it is automatically satisfied for all points with $\left(x_{2}-x_{1}\right)\left(y_{2}-y_{1}\right)>0$. Löwner's characterization of matrix monotonicity is highly sophisticated even in the case of only two by two matrices, and it is outside the scope of the present paper to give the proof. Although the area has received much attention there are no good references other than the (difficult to read) original article. This is because later authors have concentrated on alternative ways of characterizing matrix monotonicity resulting in a situation where condition (1), even among specialists, is not well known.

Corollary 2.2. Let I be an open interval.

(i) The set of utility functions defined in I with decreasing relative risk premium is a convex cone.

(ii) If $u_{1}$ and $u_{2}$ are utility functions with decreasing relative risk premium defined in open intervals $I_{1}$ and $I_{2}$ and $u_{1}\left(I_{1}\right) \subseteq I_{2}$, then the composed utility function $u(x)=u_{2}\left(u_{1}(x)\right)$ has decreasing relative risk premium.

Proof. The two assertions are trivial consequences of 2-monotonicity. Indeed, if $A \leq B$ are Hermitian two by two matrices with eigenvalues in $I$ and $u_{1}$ and $u_{2}$ are 2-monotone, then

$$
\left(u_{1}+u_{2}\right)(A)=u_{1}(A)+u_{2}(A) \leq u_{1}(B)+u_{2}(B)=\left(u_{1}+u_{2}\right)(B)
$$

which shows that $u_{1}+u_{2}$ is 2 -monotone and hence have decreasing relative risk premium. Similarly, $u_{1}(A) \leq u_{1}(B)$ and hence $u_{2}\left(u_{1}(A)\right) \leq u_{2}\left(u_{1}(B)\right)$ showing that $u_{1} \circ u_{2}$ is 2-monotone and therefore has decreasing relative risk premium.

QED

Corollary 2.3. Let u be a utility function defined in an open interval I with decreasing relative risk premium, then

(i) $u$ is automatically continuously differentiable.

(ii) If the derivative $u^{\prime}(x)=0$ in some point $x \in I$, then $u$ is constant.

Proof. The first item is a non-trivial part of Löwner's theorem [5]. Since $u$ is continuously differentiable we may in condition (1) let $y_{1}$ tend to $x_{1}$ and let $y_{2}$ tend to $x_{2}$ to obtain

$$
u^{\prime}\left(x_{1}\right) u^{\prime}\left(x_{2}\right) \geq\left[x_{1}, x_{2}\right]_{u}^{2} \geq 0 .
$$

Thus, if $u^{\prime}\left(x_{1}\right)=0$ then $u\left(x_{2}\right)=u\left(x_{1}\right)$ for all $x_{2}$ in $I$. 
The necessary condition in equation (2) may be reformulated as positive definiteness of the matrix

$$
\left(\begin{array}{cc}
u^{\prime}\left(x_{1}\right) & {\left[x_{1}, x_{2}\right]_{u}} \\
{\left[x_{1}, x_{2}\right]_{u}} & u^{\prime}\left(x_{2}\right)
\end{array}\right) \quad x_{1}, x_{2} \in I .
$$

For a continuously differentiable function $u$ this condition also implies 2monotonicity [5] and therefore decreasing relative risk premium.

A utility function with decreasing relative risk premium is automatically continuously differentiable, but it may not be twice differentiable. However, we may in most applications assume that it is even infinitely many times differentiable. Indeed, let $\varphi$ be a positive and even $C^{\infty}$-function defined in the real axis, vanishing outside the closed interval $[-1,1]$ and normalized such that

$$
\int_{-1}^{1} \varphi(x) d x=1
$$

For any locally integrable function $u$ defined in an open interval $(a, b)$ we form its regularization

$$
u_{\epsilon}(t)=\frac{1}{\epsilon} \int_{a}^{b} \varphi\left(\frac{t-s}{\epsilon}\right) u(s) d s \quad t \in \mathbf{R}
$$

for small $\epsilon>0$, and realize that it is infinitely many times differentiable. For $t \in(a+\epsilon, b-\epsilon)$ we may also write

$$
u_{\epsilon}(t)=\int_{-1}^{1} \varphi(s) u(t-\epsilon s) d s .
$$

If $u$ is continuous, then $u_{\epsilon}$ converges uniformly to $u$ on any compact subinterval of $(a, b)$. If $u$ is a utility function with decreasing relative risk premium, then it is 2-monotone in $(a, b)$ and $u_{\epsilon}$ is 2-monotone in the slightly smaller interval $(a+\epsilon, b-\epsilon)$. In conclusion, $u$ is the uniform limit in any compact subinterval of $(a, b)$ of the utility functions $u_{\epsilon}$ which are infinitely many times differentiable and have decreasing relative risk premium.

We can now give a concrete description of the set of utility functions representing preferences with decreasing relative risk premium (the proof may be found in the appendix).

Theorem 2.4. Let $u$ be a strictly increasing three times continuously differentiable function defined in an open interval I. The following conditions are equivalent:

(i) u has decreasing relative risk premium. 
(ii) The matrix

$$
\left(\begin{array}{cc}
u^{\prime}(x) & \frac{u^{\prime \prime}(x)}{2} \\
\frac{u^{\prime \prime}(x)}{2} & \frac{u^{\prime \prime \prime}(x)}{6}
\end{array}\right)
$$

is positive semi-definite for each $x \in I$.

(iii) The derivative $u^{\prime}$ can be written on the form

$$
u^{\prime}(x)=\frac{1}{c(x)^{2}} \quad x \in I
$$

where $c$ is a positive concave function.

It is an easy consequence of Theorem 2.4 (iii) that the utility functions mentioned in the introduction represent decreasing relative risk premium. Indeed, if we for each of these functions calculate the corresponding function $c(x)$ such that $u^{\prime}(x)=c(x)^{-2}$,

$$
\begin{array}{rlrl}
u(x) & =x^{\alpha} & c(x) & =\alpha^{-1 / 2} x^{(1-\alpha) / 2} \\
u(x) & =\frac{x}{x+\lambda} & c(x) & =\lambda^{-1 / 2}(x+\lambda) \\
u(x) & =\log x & c(x) & =x^{1 / 2},
\end{array}
$$

then we realize that $c(x)$ in each case is positive and concave. But we also realize that utility functions which are not concave may have the same property. Consider for example the function

$$
u(x)=\frac{1}{1-x} \quad 0<x<1,
$$

then $u^{\prime}(x)=c(x)^{-2}$ where $c(x)=1-x$ is positive and concave in $(0,1)$. The increasing function $u(x)$ is therefore both convex and represents decreasing relative risk premium.

Finally, the utility function $u(x)=1-e^{-x}$ is increasing, concave and has constant absolute risk aversion, but does not represent decreasing absolute risk premium. Indeed, $u^{\prime}(x)=c(x)^{-2}$ and the function $c(x)=e^{x / 2}$ is not concave.

\subsection{In the small and in the large}

The notion of decreasing relative risk premium depends on the comparison of utility values for different and possibly wildly separated levels of wealth, 
and it is therefore of a global nature by construction. A priory it is far from obvious whether it would be possible to piece together utility functions, which each has decreasing relative risk premium in small but overlapping intervals, and in this way obtain a function with decreasing relative risk premium in the union of the intervals.

Corollary 2.5. Decreasing relative risk premium in the small implies decreasing relative risk premium in the large.

Proof. Consider a function $u$ defined in the union of two overlapping open intervals $I_{1}$ and $I_{2}$ such that the restrictions $\left.u\right|_{I_{1}}$ and $\left.u\right|_{I_{2}}$ have decreasing relative risk premium. We want to prove that $u$ necessarily has decreasing relative risk premium also in the union $I_{1} \cup I_{2}$. Possibly by first taking its regularization we may assume that $u$ is three (or infinitely many) times continuously differentiable. If $u^{\prime}(x)=0$ for any $x$ in the union $I_{1} \cup I_{2}$ then $u$ is a constant function. We may therefore also assume that $u$ is strictly increasing. The statement is now a consequence of item (ii) in Theorem 2.4.

Consider now a utility function $u$ defined in an open interval $I$ such that $u$ has decreasing relative risk premium in a (possibly very small) neighborhood of each point $x \in I$. Consider a fixed $x$ in $I$ and let $J$ be the union of all open intervals in $I$ containing $x$ and such that $u$ has decreasing relative risk premium in each of the intervals. It follows by the preceding argument that $u$ has decreasing relative risk premium in $J$. If $J$ is a proper subset of $I$ there is a $y \in J$ which is a boundary point of $J$ and a small open interval containing $y$ in which $u$ has decreasing relative risk premium. Repeating the argument we may thus conclude that $u$ has decreasing relative risk premium in a larger interval than $J$ contradicting the construction of $J$. Therefore $J=I$ and $u$ has decreasing relative risk premium in $I$.

QED

Corollary 2.6. Let $u$ be a function with decreasing relative risk premium in the interval $\left(x_{0}, \infty\right)$ for some $x_{0} \in \mathbf{R}$. Then $u$ is concave. If in addition $u$ is three times continuously differentiable, then the representing function $c$ in Theorem 2.4 (iii) is increasing.

Proof. We may by possibly considering its regularization assume that $u$ is three times continuously differentiable. The derivative then has the form

$$
u^{\prime}(x)=\frac{1}{c(x)^{2}}
$$

for some positive concave function $c$. But a positive concave function defined on $\left(x_{0}, \infty\right)$ can not decrease in any point, since otherwise it would, because 
of concavity, become negative for large values of $x \in\left(x_{0}, \infty\right)$. Therefore $c^{\prime}(x) \geq 0$ and

$$
u^{\prime \prime}(x)=-2 \frac{c^{\prime}(x)}{c(x)^{3}} \leq 0
$$

showing that $u$ is concave.

QED

It is remarkable that decreasing relative risk premium, which is a measure of risk taking, implies risk aversion on infinite intervals $\left(x_{0}, \infty\right)$. The reason is clear from the above proof. If $u(x)$ were not concave everywhere, then $c^{\prime}(x)$ would be negative for some $x$ and $c(x)$, which is concave, would then go to zero in a bounded interval. The utility function would consequently tend asymptotically to infinity as $x$ approached some finite value. In other words, if the decreasing relative risk premium were not tempered by risk aversion, then the utility would increase towards infinity for finite levels of wealth.

Corollary 2.7. Let $u$ be a three times continuously differentiable function representing decreasing relative risk premium in the interval $\left(x_{0}, \infty\right)$ for some $x_{0} \in \mathbf{R}$.

(i) The Arrow-Pratt measure of absolute risk aversion $-u^{\prime \prime}(x) / u^{\prime}(x)$ is a decreasing function in $x$ and tends to zero as $x$ approaches infinity.

(ii) The measure of relative risk aversion, $-x u^{\prime \prime}(x) / u^{\prime}(x)$, is for $x \geq 0$ an increasing function of $x$.

Proof. The derivative of the absolute risk aversion

$$
\frac{d}{d x}\left(-\frac{u^{\prime \prime}(x)}{u^{\prime}(x)}\right)=\frac{u^{\prime \prime}(x)^{2}-u^{\prime \prime \prime}(x) u^{\prime}(x)}{u^{\prime}(x)^{2}}
$$

is negative if and only if $u^{\prime}(x) u^{\prime \prime \prime}(x) \geq u^{\prime \prime}(x)^{2}$. But since $u$ satisfies the even stronger condition

$$
u^{\prime}(x) u^{\prime \prime \prime}(x) \geq \frac{3}{2} u^{\prime \prime}(x)^{2} \quad x \in I
$$

cf. Theorem 2.4 (ii), we derive that the absolute risk aversion is decreasing in $x$. The absolute risk aversion may be written on the form

$$
-\frac{u^{\prime \prime}(x)}{u^{\prime}(x)}=\frac{2 c^{\prime}(x)}{c(x)}
$$

where we used Theorem $2.4(\mathrm{iii})$, and we note that it is non-negative by Corollary 2.6. The derivative $c^{\prime}(x)$ is decreasing since $c(x)$ is concave. The 
absolute risk aversion therefore tends to zero if $c(x)$ goes to infinity. If on the other hand $c(x)$ is bounded, then the derivative $c^{\prime}(x)$ goes to zero as $x$ approaches infinity, and we obtain the same conclusion.

The second statement is obtained by considering the derivative of the relative risk aversion

$$
\frac{d}{d x}\left(\frac{2 x c^{\prime}(x)}{c(x)}\right)=2 \frac{c^{\prime}(x)-x c^{\prime \prime}(x)}{c(x)^{2}}
$$

which for $x \geq 0$ is non-negative since $c$ is increasing and concave.

QED

\section{Preferences on lotteries}

Suppose that a decision maker with utility function $u$ has to decide between lotteries $\tilde{x}$ and $\tilde{y}$ with outcomes $\left(x_{1}, x_{2}\right)$ and $\left(y_{1}, y_{2}\right)$ respectively. Then $\tilde{y}$ is preferred if the expected utilities satisfy

$$
E_{u}(\tilde{x}, p)=p u\left(x_{1}\right)+(1-p) u\left(x_{2}\right) \leq q u\left(y_{1}\right)+(1-q) u\left(y_{2}\right)=E_{u}(\tilde{y}, q),
$$

where $p$ is the probability of outcome $x_{1}$ in $\tilde{x}$ and $q$ is the probability of outcome $y_{1}$ in $\tilde{y}$. The only case difficult to analyze is when the outcomes are intertwined like $x_{1}<y_{1}<x_{2}<y_{2}$. We then introduce the numbers

$$
a=\sqrt{\frac{\left(x_{2}-y_{1}\right)\left(y_{2}-x_{1}\right)}{\left(x_{2}-x_{1}\right)\left(y_{2}-y_{1}\right)}}, \quad b=\sqrt{\frac{\left(y_{1}-x_{1}\right)\left(y_{2}-x_{2}\right)}{\left(x_{2}-x_{1}\right)\left(y_{2}-y_{1}\right)}}
$$

and note that they only depend on the possible outcomes.

Lemma 3.1. Let $x_{1}<y_{1}<x_{2}<y_{2}$ be real numbers. The mapping

$$
q(p)=p a^{2}+2 p^{1 / 2}(1-p)^{1 / 2} a b+(1-p) b^{2},
$$

where $a$ and $b$ are defined in (4), maps the unit interval $[0,1]$ into itself. It is minimal in the sense that there exists a probability $p \in[0,1]$ such that the expected outcomes $E(\tilde{x}, p)$ and $E(\tilde{y}, q(p))$ of the two lotteries are equal. Furthermore, the expected outcomes satisfy

$$
E(\tilde{x}, p) \leq E(\tilde{y}, q)
$$

for every $p \in[0,1]$ and $q \in[0, q(p)]$. 
Proof. A simple calculation shows that $a^{2}+b^{2}=1$, therefore the matrix

$$
Q=\left(\begin{array}{cc}
a & b \\
-b & a
\end{array}\right)
$$

is orthogonal, that is $Q^{*}=Q^{-1}$. The vector given by

$$
Q\left(\begin{array}{c}
p^{1 / 2} \\
(1-p)^{1 / 2}
\end{array}\right)=\left(\begin{array}{c}
a p^{1 / 2}+b(1-p)^{1 / 2} \\
b p^{1 / 2}-a(1-p)^{1 / 2}
\end{array}\right)
$$

is consequently a unit vector, and since

$$
\left(a p^{1 / 2}+b(1-p)^{1 / 2}\right)^{2}=p a^{2}+2 p^{1 / 2}(1-p)^{1 / 2} a b+(1-p) b^{2}=q(p)
$$

we obtain $0 \leq q(p) \leq 1$. We obtain by tedious calculations

$$
Q^{-1}\left(\begin{array}{cc}
y_{1} & 0 \\
0 & y_{2}
\end{array}\right) Q-\left(\begin{array}{cc}
x_{1} & 0 \\
0 & x_{2}
\end{array}\right)=\left(\begin{array}{cc}
\alpha^{2} & \alpha \beta \\
\alpha \beta & \beta^{2}
\end{array}\right)
$$

where

$$
\alpha=\sqrt{\frac{\left(y_{1}-x_{1}\right)\left(y_{2}-x_{1}\right)}{\left(x_{2}-x_{1}\right)}}, \quad \beta=\sqrt{\frac{\left(x_{2}-y_{1}\right)\left(y_{2}-x_{2}\right)}{\left(x_{2}-x_{1}\right)}} .
$$

Since the right hand side of (6) is positive semi-definite, we have for arbitrary $p \in[0,1]$ that

$$
\begin{aligned}
E(\tilde{x}, p) & =p x_{1}+(1-p) x_{2} \\
& =\left(\left(\begin{array}{cc}
x_{1} & 0 \\
0 & x_{2}
\end{array}\right)\left(\begin{array}{c}
p^{1 / 2} \\
(1-p)^{1 / 2}
\end{array}\right) \mid\left(\begin{array}{c}
p^{1 / 2} \\
(1-p)^{1 / 2}
\end{array}\right)\right) \\
& \leq\left(Q^{-1}\left(\begin{array}{cc}
y_{1} & 0 \\
0 & y_{2}
\end{array}\right) Q\left(\begin{array}{c}
p^{1 / 2} \\
(1-p)^{1 / 2}
\end{array}\right) \mid\left(\begin{array}{c}
p^{1 / 2} \\
(1-p)^{1 / 2}
\end{array}\right)\right) \\
& =\left(\left(\begin{array}{cc}
y_{1} & 0 \\
0 & y_{2}
\end{array}\right) Q\left(\begin{array}{c}
p^{1 / 2} \\
(1-p)^{1 / 2}
\end{array}\right) \mid Q\left(\begin{array}{c}
p^{1 / 2} \\
(1-p)^{1 / 2}
\end{array}\right)\right) \\
& =q(p) y_{1}+(1-q(p)) y_{2} \\
& =E(\tilde{y}, q(p)) \leq E(\tilde{y}, q), \quad 0 \leq q \leq q(p) .
\end{aligned}
$$

Finally, for $p=\alpha\left(\alpha^{2}+\beta^{2}\right)^{-1 / 2}$ we obtain $E(\tilde{x}, p)=E(\tilde{y}, q(p))$.

The proof of the next theorem may be found in the appendix. 
Theorem 3.2. Let $u$ be a utility function defined in an open interval I, and let $x_{1}<y_{1}<x_{2}<y_{2}$ be intertwined outcomes in two lotteries $\tilde{x}$ and $\tilde{y}$. If $u$ has decreasing relative risk premium, then the expected utilities

$$
E_{u}(\tilde{x}, p) \leq E_{u}(\tilde{y}, q) \quad 0 \leq q \leq q(p)
$$

for every $p \in[0,1]$, where $q(p)$ is the function defined in Lemma 3.1. If on the other hand a decision maker share these preferences on lotteries, then he necessarily has decreasing relative risk premium.

A decision maker with decreasing relative risk premium makes the same decisions as a risk neutral decision maker would do when confronted with the choices in the preceding theorem, cf. Lemma 3.1. This is not surprising since a risk neutral decision maker has decreasing relative risk premium.

Definition 3.3. We say that a decision maker is partially risk neutral, if he prefers $(\tilde{y}, q)$ to $(\tilde{x}, p)$ for every $p \in[0,1]$ and $q \in[0, q(p)]$ for all binary lotteries $(\tilde{x}, p)$ and $(\tilde{y}, q)$ with intertwined outcomes $x_{1}<y_{1}<x_{2}<y_{2}$, where $q(p)$ is the function defined in Lemma 3.1.

Note that the expected outcomes of the lotteries in the preceding definition satisfy $E(\tilde{x}, p) \leq E(\tilde{y}, q)$. We have thus established:

Theorem 3.4. A decision maker is partially risk neutral if and only if his preferences represent decreasing relative risk premium.

\subsection{Examples}

Heterogeneous decision makers with possibly very different utility functions, but of the form mentioned in the introduction, nevertheless share preferences on the risky lotteries exhibited in this section. In the following example we set $x_{1}=1, y_{1}=2, x_{2}=3$ and $y_{2}=4$. We obtain $a=\sqrt{3} / 2, b=1 / 2$ and calculate the probability map

$$
q(p)=\frac{1}{4}+\frac{p}{2}+\frac{\sqrt{3}}{2} p^{1 / 2}(1-p)^{1 / 2} .
$$

We consider two decision makers with utility functions $u_{1}(x)=x^{1 / 2}$ and $u_{2}(x)=x^{4 / 10}$. The expected utility of $(\tilde{x}, p)$ as a function of $p$ is for the two decision makers indicated by the two straight lines in the diagram. Since $u_{2} \geq$ $u_{1}$ in the four outcomes, we realize that the upper straight line represents the expected utility $E_{u_{2}}(\tilde{x}, p)$. We note that, although the decision makers are heterogeneous, they have for all $p \in[0,1]$ the same preferences on the lotteries 
$(\tilde{x}, p)$ and $(\tilde{y}, q(p))$. In fact, they share these preferences with all decision makers, some risk averse and some risk lovers, with decreasing relative risk premium in an open interval containing the four outcomes.

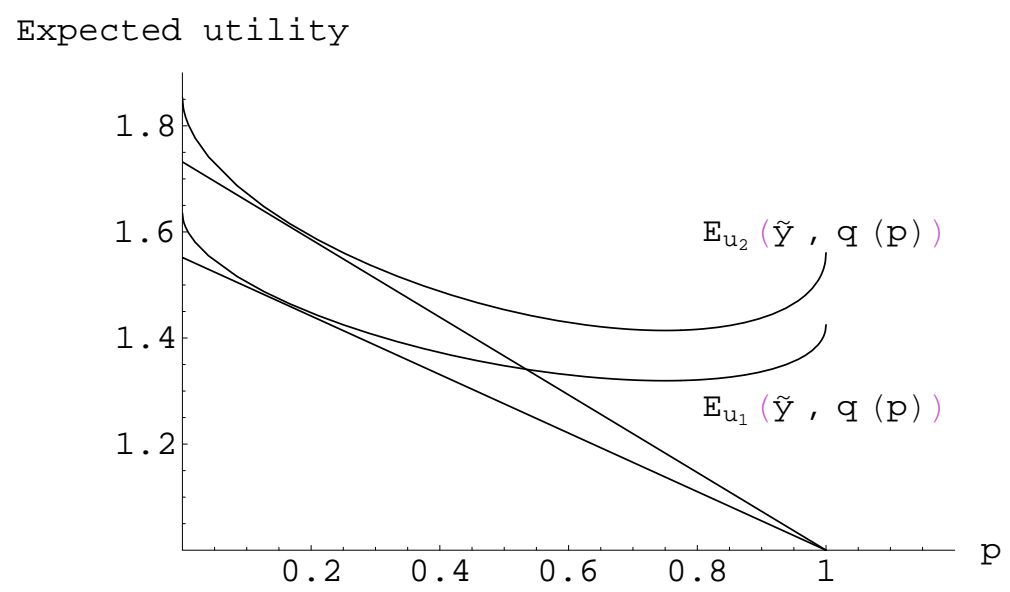

We learned in Theorem 3.2 that a decision maker may share the preferences on the type of risky lotteries considered in this section with one (and then with all) decision makers with decreasing relative risk premium, if and only if he himself has decreasing relative risk premium.

Let us now consider a decision maker with a utility function

$$
u_{3}(x)=1-\exp (2-x)
$$

with constant absolut risk aversion. It does not represent decreasing relative risk premium, and this is reflected in the next diagram.

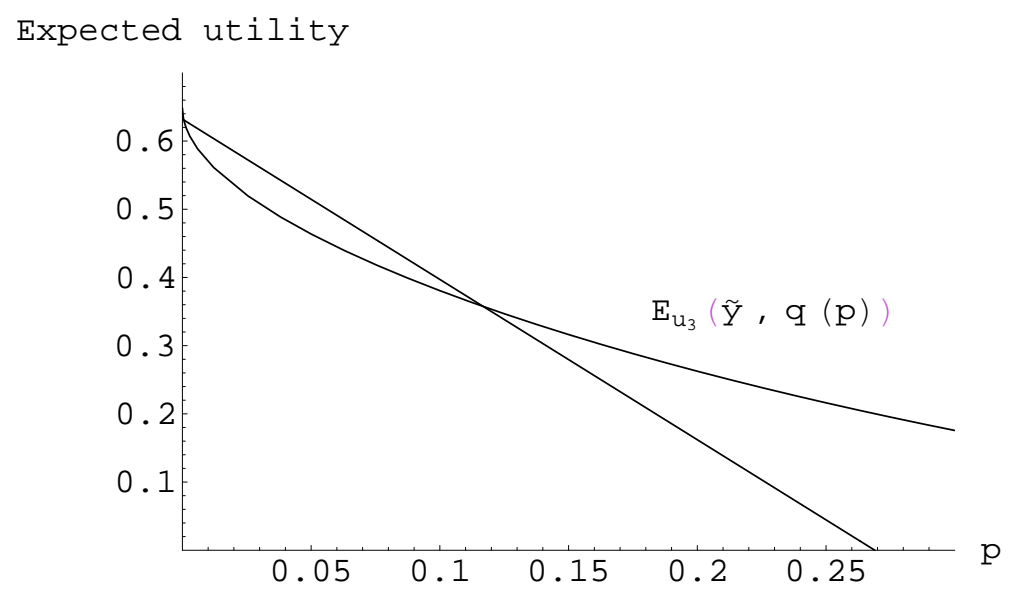


For small values of $p$ the third decision maker prefers $(\tilde{x}, p)$ for $(\tilde{y}, q(p))$, thus disagreeing with the first two decision makers.

We have shown that preferences with decreasing relative risk premium may be equivalently expressed by preferences on risky lotteries. It seems very difficult to obtain this equivalence without first characterizing the corresponding class of utility functions.

\section{Appendix}

Proof of Theorem 2.4: We rely heavily on the work of Dobsch [1] and Donoghue [2]. Firstly, we remind that higher ordens divided differences (in mutually distinct points) are defined recursively by setting

$$
\begin{aligned}
{\left[x_{1}, x_{2}, x_{3}\right]_{u} } & =\frac{\left[x_{1}, x_{2}\right]_{u}-\left[x_{2}, x_{3}\right]_{u}}{x_{1}-x_{3}} \\
{\left[x_{1}, x_{2}, x_{3}, x_{4}\right]_{u} } & =\frac{\left[x_{1}, x_{2}, x_{3}\right]_{u}-\left[x_{2}, x_{3}, x_{4}\right]_{u}}{x_{1}-x_{4}} .
\end{aligned}
$$

They are symmetric functions in the arguments $x_{1}, x_{2}$ etc. and if $u$ is three times continuously differentiable, then the second and third order divided differences above may be extended to continuous functions. The mean value theorem for divided difference implies that

$$
[x, x, x]_{u}=\frac{u^{\prime \prime}(x)}{2 !} \quad \text { and } \quad[x, x, x, x]_{u}=\frac{u^{\prime \prime \prime}(x)}{3 !}
$$

for arbitrary $x \in I$.

$(i) \Rightarrow(i i)$ :

If $u$ represents decreasing relative risk premium, then the determinant

$$
D=\operatorname{det}\left(\begin{array}{ll}
{\left[x_{1}, x_{1}\right]_{u}} & {\left[x_{1}, x_{2}\right]_{u}} \\
{\left[x_{1}, x_{2}\right]_{u}} & {\left[x_{2}, x_{2}\right]_{u}}
\end{array}\right) \geq 0
$$

where we write $[x, x]_{u}=u^{\prime}(x)$. Let $x_{1} \neq x_{2}$ and subtract the first column from the second. By using the recursive property of divided differences we obtain

$$
D=\left(x_{2}-x_{1}\right) \operatorname{det}\left(\begin{array}{ll}
{\left[x_{1}, x_{1}\right]_{u}} & {\left[x_{2}, x_{1}, x_{1}\right]_{u}} \\
{\left[x_{1}, x_{2}\right]_{u}} & {\left[x_{2}, x_{2}, x_{1}\right]_{u}}
\end{array}\right) \text {. }
$$

Next we subtract the first row from the second row in the new matrix and obtain

$$
D=\left(x_{2}-x_{1}\right)^{2} \operatorname{det}\left(\begin{array}{cc}
{\left[x_{1}, x_{1}\right]_{u}} & {\left[x_{1}, x_{1}, x_{2}\right]_{u}} \\
{\left[x_{1}, x_{2}, x_{1}\right]_{u}} & {\left[x_{1}, x_{2}, x_{1}, x_{2}\right]_{u}}
\end{array}\right) .
$$


By letting $x_{1}$ and $x_{2}$ tend to $x$ we obtain

$$
\operatorname{det}\left(\begin{array}{cc}
u^{\prime}(x) & \frac{u^{\prime \prime}(x)}{2} \\
\frac{u^{\prime \prime}(x)}{2} & \frac{u^{\prime \prime \prime}(x)}{3 !}
\end{array}\right) \geq 0 .
$$

Since $u^{\prime}(x)>0$ we realize that $u^{\prime \prime \prime}(x)$ cannot be negative. All three principal determinants in the matrix are thus non-negative, and this implies (ii). $($ ii $) \Rightarrow($ iii $)$ : Put $c(x)=u^{\prime}(x)^{-1 / 2}$ for $x \in I$. Then $c$ is a positive function and $u^{\prime}(x)=c(x)^{-2}$. By differentiation we obtain $u^{\prime \prime}(x)=-2 c(x)^{-3} c^{\prime}(x)$ and

$$
u^{\prime \prime \prime}(x)=6 c(x)^{-4} c^{\prime}(x)^{2}-2 c(x)^{-3} c^{\prime \prime}(x) .
$$

The determinant

$$
\frac{1}{6} u^{\prime}(x) u^{\prime \prime \prime}(x)-\frac{1}{4} u^{\prime \prime}(x)^{2}
$$

is non-negative by $(i i)$, thus inserting the derivatives we obtain

$$
\begin{aligned}
& \frac{1}{6} c(x)^{-2}\left(6 c(x)^{-4} c^{\prime}(x)^{2}-2 c(x)^{-3} c^{\prime \prime}(x)\right)-\frac{1}{4}\left(-2 c(x)^{-3} c^{\prime}(x)\right)^{2} \\
& =-\frac{1}{3} c(x)^{-5} c^{\prime \prime}(x) \geq 0,
\end{aligned}
$$

hence $c^{\prime \prime}(x) \leq 0$ for every $x \in I$ and $c$ is concave.

$($ iii $) \Rightarrow(i)$ : First order divided differences may trivially be expressed by

$$
\left[x_{1}, x_{2}\right]_{u}=\int_{0}^{1} u^{\prime}\left((1-t) x_{1}+t x_{2}\right) d t .
$$

This is a small part of the Hermite formulae [3]. By using (iii) and the concavity of $c$ we obtain

$$
\begin{aligned}
{\left[x_{1}, x_{2}\right]_{u} } & =\int_{0}^{1} \frac{1}{c\left((1-t) x_{1}+t x_{2}\right)^{2}} d t \\
& \leq \int_{0}^{1} \frac{1}{\left((1-t) c\left(x_{1}\right)+t c\left(x_{2}\right)\right)^{2}} d t \\
& =-\int_{0}^{1} g^{\prime}\left((1-t) c\left(x_{1}\right)+t c\left(x_{2}\right)\right) d t \\
& =-\left[c\left(x_{1}\right), c\left(x_{2}\right)\right]_{g}
\end{aligned}
$$

where we set $g(x)=x^{-1}$ and used the Hermite formula once more. But since

$$
-\left[c\left(x_{1}\right), c\left(x_{2}\right)\right]_{g}=\frac{1}{c\left(x_{1}\right) c\left(x_{2}\right)}=\sqrt{u^{\prime}\left(x_{1}\right) u^{\prime}\left(x_{2}\right)}
$$


we obtain $u^{\prime}\left(x_{1}\right) u^{\prime}\left(x_{2}\right) \geq\left[x_{1}, x_{2}\right]_{u}^{2}$. But this is the local condition (3) for 2-monotonicity. Therefore $u$ represents decreasing relative risk premium.

QED

Proof of Theorem 3.2: Since by Theorem 2.1 the utility function $u$ is 2monotone, we may apply it in the matrix inequality

$$
\left(\begin{array}{cc}
x_{1} & 0 \\
0 & x_{2}
\end{array}\right) \leq Q^{-1}\left(\begin{array}{cc}
y_{1} & 0 \\
0 & y_{2}
\end{array}\right) Q
$$

derived from (6) and obtain

$$
\left(\begin{array}{cc}
u\left(x_{1}\right) & 0 \\
0 & u\left(x_{2}\right)
\end{array}\right) \leq Q^{-1}\left(\begin{array}{cc}
u\left(y_{1}\right) & 0 \\
0 & u\left(y_{2}\right)
\end{array}\right) Q
$$

Consequently,

$$
\begin{aligned}
E_{u}(\tilde{x}, p) & =p u\left(x_{1}\right)+(1-p) u\left(x_{2}\right) \\
& =\left(\left(\begin{array}{cc}
u\left(x_{1}\right) & 0 \\
0 & u\left(x_{2}\right)
\end{array}\right)\left(\begin{array}{c}
p^{1 / 2} \\
(1-p)^{1 / 2}
\end{array}\right) \mid\left(\begin{array}{c}
p^{1 / 2} \\
(1-p)^{1 / 2}
\end{array}\right)\right) \\
& \leq\left(Q^{-1}\left(\begin{array}{cc}
u\left(y_{1}\right) & 0 \\
0 & u\left(y_{2}\right)
\end{array}\right) Q\left(\begin{array}{c}
p^{1 / 2} \\
(1-p)^{1 / 2}
\end{array}\right) \mid\left(\begin{array}{c}
p^{1 / 2} \\
(1-p)^{1 / 2}
\end{array}\right)\right) \\
& =q(p) u\left(y_{1}\right)+(1-q(p)) u\left(y_{2}\right) \\
& =E_{u}(\tilde{y}, q(p)) \leq E_{u}(\tilde{y}, q), \quad 0 \leq q \leq q(p),
\end{aligned}
$$

where we used that $Q$ transforms the probability distribution $(p, 1-p)$ into the distribution $(q(p), 1-q(p))$.

Suppose on the other hand that a decision maker with increasing utility function $u$ defined in an open interval $I$ prefers the lotteries $(\tilde{y}, q(p))$ for $(\tilde{x}, p)$ for arbitrary outcomes $x_{1}<y_{1}<x_{2}<y_{2}$ in $I$ and every $p \in[0,1]$, where $q(p)$ is defined as in Lemma 3.1. Let now $A$ and $B$ be Hermitian two by two matrices with eigenvalues in $I$ and suppose

$$
B=A+c P,
$$

where $c>0$ and $P$ is the orthogonal projection on a single unit vector. Donoghue proved [2, Chapter VI] that if $x_{1}, x_{2}$ are the eigenvalues of $A$ and $y_{1}, y_{2}$ are the eigenvalues of $B$, both written in increasing order, then they are intertwined $x_{1}<y_{1}<x_{2}<y_{2}$ and the equation $B-A=c P$ may be uniquely written as in equation (6). The decision maker's preferences on lotteries therefore imply that $u(A) \leq u(B)$. Let finally $A$ and $B$ be arbitrary 
Hermitian two by two matrices with eigenvalues in $I$ and suppose that $A \leq B$. By the spectral theorem we may write

$$
A \leq C \leq B,
$$

where the differences $C-A$ and $B-C$ both are positive multiples of onedimensional projections. Repeated application of the previous result thus entails $u(A) \leq u(B)$. But this means that $u$ is 2 -monotone and the decision maker therefore has decreasing relative risk premium.

QED

\section{References}

[1] O. Dobsch. Matrixfunktionen beschränkter schwankung. Math. Z., 43:353-388, 1937.

[2] W. Donoghue. Monotone matrix functions and analytic continuation. Springer, Berlin, Heidelberg, New York, 1974.

[3] Ch. Hermite. Sur la formule d'interpolation de Lagrange. Journal für die Reine und Angewandte Mathematik, 84:70-79, 1878.

[4] M.S. Kimball. Standard risk aversion. Econometrica, 61:589-611, 1993.

[5] K. Löwner. Über monotone Matrixfunktionen. Math. Z., 38:177-216, 1934.

[6] L.T. Nielsen. Monotone risk aversion. Economique Theory, 25:203-215, 2005.

[7] J. Pratt. Risk aversion in the small and in the large. Econometrica, 32:122-136, 1967.

[8] J. Quiggin and R.G. Chambers. Risk premiums and benefit measures for generalizedexpected-utility theories. Journal of risk and uncertainty, 17:121-137, 1998.

[9] M. Rabin. Risk aversion and expected-utility theory: A calibration theorem. Econometrica, 68:1281-1292, 2000.

[10] S.A. Ross. Some stronger measures of risk aversion in the small and the large with applications. Econometrica, 49:621-638, 1981.

[11] M.E. Yaari. Some remarks on the measures of risk aversion and on their uses. Journal of Economic theory, 1:315-329, 1969.

Frank Hansen: Department of Economics, University of Copenhagen, Studiestraede 6, DK-1455 Copenhagen K, Denmark. 